\title{
Tratamento da Hipersensibilidade dentinária nas lesões cervicais não cariosas:
}

\section{revisão da literatura}

\author{
Treatment of dentin hypersensitivity in non-carious cervical lesions: a review of the literature \\ Tratamiento de la hipersensibilidad dentinaria en lesiones cervicales no cariadas: una revisión de la
}

literatura

Recebido: 02/11/2021 | Revisado: 09/11/2021 | Aceito: 11/11/2021 | Publicado: 20/11/2021

\author{
Débora Rezende Miranda \\ ORCID: https://orcid.org/0000-0001-8933-2148 \\ Centro Universitário de Patos de Minas, Brasil \\ E-mail: deborarm@unipam.edu.br \\ Carolina Silva Pereira \\ ORCID: https://orcid.org/0000-0003-2020-3932 \\ Centro Universitário de Patos de Minas, Brasil \\ E-mail: carolinasp@unipam.edu.br \\ Lorene Queiroz Casali Reis \\ ORCID: https://orcid.org/0000-0001-5551-2255 \\ Centro Universitário de Patos de Minas, Brasil \\ E-mail: lorenepqc@unipam.edu.br
}

\begin{abstract}
Resumo
A Hipersensibilidade dentinária (HD) pode ser definida como um processo patológico que tem etiologia multifatorial e depende de estímulos em túbulos dentinários expostos supragengivais, subgengivais e abaixo de defeitos estruturais e trincas de esmalte que podem desencadear uma dor de sintomatologia aguda e de curta duração. De acordo com a teoria da Hidrodinâmica, a HD é o resultado do fluxo de fluido nos túbulos da dentina. Comumente, está associada as Lesões cervicais não cariosas e a fatores como tensão, fricção e biocorrosão, que podem atuar isoladamente ou em conjunto. O presente trabalho revisou a literatura em relação aos tratamentos para a HD em Lesões cervicais não cariosas. Foram utilizadas as palavras-chave e seus correspondentes na língua inglesa, sendo possível encontrar na literatura científica variadas técnicas de recursos terapêuticos para reduzir os sintomas da HD, contudo, a aplicação destas deve ser iniciada a partir de um minucioso diagnóstico clínico e diferencial associado a um exame físico extra-oral, clínico intra-oral e exame radiográfico detalhado, diferenciando a HD de outras patologias. O objetivo dos tratamentos são identificar e controlar os fatores causais, o material ideal para tratamento de hipersensibilidade dentinária deve ser biocompatível, de fácil aplicação, ter efeito duradouro, permanente e de ação rápida, não ser um irritante pulpar e não alterar a cor da estrutura dental. Uma combinação de instrução de higiene oral, uso de dessensibilizantes caseiros e tratamentos realizados em consultório odontológico, incluindo dessensibilizantes de uso profissional e tratamentos restauradores, são capazes de diminuir e eliminar a hipersensibilidade dentinária.
\end{abstract}

Palavras-chave: Sensibilidade da dentina; Esmalte dentário; Assistência odontológica.

\begin{abstract}
Dentin hypersensitivity (HD) can be defined as a pathological process that has a multifactorial etiology and depends on stimuli in exposed dentinal tubules supragingivally, subgingivally and below structural defects and enamel cracks that can trigger a pain of acute and short-lived symptomatology. According to the hydrodynamic theory, HD is the result of fluid flow in the dentin tubules. It is commonly associated with non-carious cervical lesions and factors such as tension, friction and biocorrosion, which may act alone or in combination. This paper reviewed the literature regarding treatments for HD in non-carious cervical lesions. It was possible to find in the scientific literature several techniques of therapeutic resources to reduce the symptoms of HD. However, the application of these resources should be initiated after a thorough clinical and differential diagnosis associated with an extra-oral physical examination, intra-oral clinical examination and detailed radiographic examination, differentiating HD from other pathologies. The objective of the treatments is to identify and control the causal factors, the ideal material for dentin hypersensitivity treatment must be biocompatible, easy to apply, have a long-lasting, permanent and fast-acting effect, not be a pulp irritant, and not alter the color of the tooth structure. A combination of oral hygiene instruction, use of home desensitizers, and treatments performed in the dental office, including professional use desensitizers and restorative treatments, are able to decrease and eliminate dentin hypersensitivity.
\end{abstract}

Keywords: Dentin sensitivity; Dental enamel; Dental care. 


\begin{abstract}
Resumen
La hipersensibilidad dental (DH) puede definirse como un proceso patológico de etiología multifactorial que depende de estímulos en los túbulos dentinarios expuestos supragingivalmente, subgingivalmente y por debajo de los defectos estructurales y las grietas del esmalte que pueden desencadenar un dolor de sintomatología aguda y de corta duración. Según la teoría hidrodinámica, la EH es el resultado del flujo de fluidos en los túbulos dentinarios. Suele asociarse a lesiones cervicales no cariadas y a factores como la tensión, la fricción y la biocorrosión, que pueden actuar solos o combinados. Este estudio revisa la literatura sobre el tratamiento de la DN en lesiones cervicales no cariadas. Fue posible encontrar en la literatura científica varias técnicas de recursos terapéuticos para reducir los síntomas de la EH. Sin embargo, su aplicación debe ser iniciada después de un minucioso diagnóstico clínico y diferencial asociado a un examen físico extraoral, examen clínico intraoral y examen radiográfico detallado, diferenciando la EH de otras patologías. El objetivo de los tratamientos es identificar y controlar los factores causales, el material ideal para tratar la hipersensibilidad dentinaria debe ser biocompatible, fácil de aplicar, tener un efecto duradero, permanente y de acción rápida, no ser irritante para la pulpa y no alterar el color de la estructura dental. Una combinación de instrucciones de higiene bucal, el uso de desensibilizadores caseros y los tratamientos realizados en el consultorio dental, incluidos los desensibilizadores profesionales y los tratamientos restauradores, son capaces de disminuir y eliminar la hipersensibilidad dentinaria.
\end{abstract}

Palabras clave: Sensibilidad de la dentina; Esmalte dental; Atención odontológica.

\title{
1. Introdução
}

A Hipersensibilidade dentinária (HD), pode ser definida como um processo patológico que tem etiologia multifatorial e depende de estímulos (físicos, químicos, osmóticos, de pressão, ou temperatura) em túbulos dentinários expostos supragengivais, subgengivais e abaixo de defeitos estruturais e trincas de esmalte que podem desencadear uma dor de sintomatologia aguda e de curta duração (Zeola; Soares \& Cunha-Cruz, 2019). Ela atinge cerca de 35\% da população mundial, afetando 1 a cada 6 pessoas, com incidência maior em indivíduos na faixa etária dos 30 anos (Gondim et al., 2011). Os dentes mais afetados pela HD são os posteriores (Nascimento et al, 2020).

De acordo com a teoria da Hidrodinâmica, a hipersensibilidade dentinária é o resultado do fluxo de fluido nos túbulos da dentina. Se induzidos estímulos de frio e evaporação, eles vão causar uma saída de fluido da dentina, já os estímulos de calor vão provocar um deslocamento de fluido em direção a polpa dentária. Após a indução desses estímulos, a dentina ativa as fibras nervosas intradentárias e causa a dor (Matias et al., 2010).

As Lesões Cervicais não cariosas (LCNC), que são caracterizadas pela perda de tecido mineralizado na região cervical da coroa dentária e superfície radicular subjacente, são uma das mais responsáveis pelo aparecimento de hipersensibilidade dentinária cervical (HSDC) (Regiani et al., 2021).

Essas lesões, apesar de serem multifatoriais, são normalmente classificadas pela sua etiologia, comumente classificadas em fricção, tensão e biocorrosão (Pieralisi, 2003). O grande risco para o desenvolvimento da HD está na associação de todos esses fatores.

A fricção é um desgaste gerado por atritos na estrutura dentária; podem ser intrínsecos (atrição: dente x dente) ou extrínsecos (abrasão: agentes abrasivos externos, como dentifrícios e cerdas de escova x dente) (Grippo; Simring; Coleman; 2012). A tensão (abfração) é a perda do tecido dental causada pela sobrecarga oclusal dos dentes resultantes da disfunção do sistema mastigatório, de alguns dentes mal posicionados, de hábitos parafuncionais e bruxismo. Já a biocorrosão é causada por fatores intrínsecos como refluxos gástricos e bulimia nervosa, e também fatores extrínsecos como o consumo excessivo de alimentos e bebidas ácidas. Todos esses fatores podem atuar separados ou em conjunto (Tolentino, 2016).

O tratamento para a HD nas LCNC deve ter início a partir do diagnóstico clínico e diferencial. Na literatura são encontradas várias formas para tentar minimizar essa sintomatologia (Regiani et al., 2021).

O objetivo dos tratamentos são identificar e controlar os fatores causais (tensão, biocorrosão e fricção). A abordagem conservadora convencional requer a utilização de agentes dessensibilizantes de aplicação tópica por profissional. Esses agentes dessensibilizantes, de acordo com a teoria da hidrodinâmica, possibilitam a diminuição ou anulação dos estímulos, através dos 
mecanismos neurais e obliteradores, então em áreas com mínimas perdas de estruturas dentárias e sintomatologia, indica-se dessensibilizantes químicos (Rosing et al, 2009). Também é indicado como tratamento, restaurações em resina composta, onde se tem áreas com extensas exposições dentinárias, e enxertos periodontais quando há presença de recessões gengivais.

Outro método bastante estudado atualmente é a fotobiomodulação, representada pela utilização de lasers sobre os tecidos. De acordo com a potência e a capacidade de interação com os tecidos, os Lasers utilizados para esses fins podem ser dividos em 2 grupos: os Lasers de Alta Potência (LAP), e os Lasers de Baixa potência (LBP) (Barros et al., 2008).

Este estudo teve como objetivo buscar evidências científicas, através de uma revisão narrativa de literatura, sobre a efetividade desses tratamentos citados acima em reduzir ou eliminar a Hipersensibilidade dentinária nas lesões cervicais não cariosas.

\section{Metodologia}

O presente trabalho consiste em uma revisão de literatura de caráter narrativo, natureza básica pura, objetivo descritivo e enquadramento na modalidade bibliográfica, sendo o estudo fundamentado no método qualitativo. Rother (2007) afirma que a revisão narrativa é apropriada para descrever ou discutir o "estado da arte" de determinado assunto, sob o ponto de vista teórico ou contextual. As fontes utilizadas foram livros, artigos científicos de revisão sistemática, revisão crítica, monografias e teses nas línguas inglesa e portuguesa e revistas eletrônicas. Foi realizado a leitura dos artigos que possuíam concreta relação com o tema deste trabalho retirando informações que comporam esta revisão. Foram utilizados para a busca a biblioteca local do Centro Universitário de Patos de Minas - MG e os repositórios on-line Scielo, PubMed e Medline usando como palavras-chave "Sensibilidade da Dentina”, "Esmalte Dentário" e "Assistência Odontológica” e seus correspondentes na língua inglesa.

\section{Resultados}

\subsection{Lesões Cervicais Não Cariosas e a Hipersensibilidade Dentinária}

Com a crescente expectativa de vida e o envelhecimento, as pessoas tem sustentado a sua dentição permanente durante quase toda a vida (Costa, 2007). Assim, os elementos dentários são expostos por um período maior à fatores etiológicos que os indivíduos jovens, causando injúrias provenientes da perda progressiva de elementos importantes, elevando consideravelmente a casuística de lesões cervicais na clínica odontológica (Marochi \& Queiroz, 2001).

Divididas em lesões cariosas e não cariosas, as patologias cervicais quando existentes promovem perdas irreversíveis na estrutura dentária, ou seja, perde-se tecido mineralizado na região cervical da coroa dentária e na superfície radicular subjacente por meio de um processo não relacionado à cárie (Santos et al., 2013). As lesões cariosas podem ter origem no envolvimento de microrganismos, enquanto as lesões não cariosas possuem etiologia diversificada e geralmente, não possuem influência bacteriana (Grippo; Simring \& Schreiner, 2004). Nesse sentido, segundo Kliemann (2002), estas lesões são classificadas em tensão (abfração), fricção (atrito) e biocorrosão.

As lesões cervicais não cariosas (LCNCs) de friç̧ão são definidas como o desgaste patológico dos tecidos duros dos dentes por forças mecânicas, isto é, ocorre por meio de um processo mecânico repetitivo que envolve objetos ou substâncias ou atrição doa próprios dentes (Esteves, 2010). De acordo com Lussi (2006), a biocorrosão dental é a perda de substância dentária mediante processos químicos, bioquímicos e eletroquímicos, de contato frequente com ácidos, causando a perda de tecido duro, além de gerar amolecimento do elemento. As lesões por tensão são causadas por forças oclusais traumáticas ou hábitos parafuncionais que causam a perda dos tecidos duros dentários (Paiva et al., 2003).

Uma condição clínica dolorosa e frequente proveniente de lesões não cariosas é a hipersensibilidade dentinária (Garone Filho, 1996). Definida como um desconforto pela exposição do material de proteção das estruturas dentárias, ou seja, o esmalte e os túbulos dentinários (Sousa et al., 2018), a hipersensibilidade dentinária (HD) tem relação com uma resposta sensível 
exacerbada pelo organismo tendo fatores causais diversos (Silva et al., 2017). Segundo Zeola, Soares \& Cunha-Cruz (2019), uma definição mais contemporânea para a hipersensibilidade dentinária (HD) conceitua que essa condição é classificada como um processo patológico que possui causa multifatorial, sendo dependente de estímulos térmicos, táteis, osmóticos e químicos permitindo que os túbulos dentinários sejam expostos em regiões supragengivais e subgengivais, além de locais que estejam abaixo de defeitos estruturais do esmalte. A partir dessa injúria, o indivíduo relata dor aguda e de curta duração.

Epidemiologicamente, a hipersensibilidade dentinária afeta 35\% da população mundial, ou seja, 1 a cada 6 pessoas possuem essa condição. Tendo uma incidência maior em indivíduos na faixa etária dos 30 anos e não há nenhuma diferença estatística entre homens e mulheres (Hotta et al., 2006), a HD pode apresentar resolução espontânea por meio da remineralização gerada pela saliva ou pela formação de dentina reacional (Rimondini; Baroni \& Carrassi, 1995).

Apesar da casuística de LCNC ser comum na clínica odontológica, há falhas na compreensão entre os profissionais acerca da causa, prevenção e os tipos de tratamentos disponibilizados (Lima et al., 2005). Diante disso, Lyttle et al. (1998) realizaram um levantamento sobre o conhecimento de um grupo de dentistas sobre a etiologia e a terapêutica clínica que deveriam ser adotadas diante das lesões que lhes foi mostrada através de imagens radiográficas. Dentre os erros encontrados, a classificação de lesões abrasivas como abfrações foi a mais prevalente, constando a escovação como fator etiológico em $81 \%$ dos participantes e $36 \%$ afirmaram que as lesões teriam origem em forças oclusais.

\subsection{Efetividade e fragilidades dos tratamentos contemporâneos para a hipersensibilidade dentinária}

É possível encontrar na literatura científica variadas técnicas de recursos terapêuticos para reduzir os sintomas da hipersensibilidade dentinária (Tonetto et al., 2012). Contudo, a aplicação destes deve ser iniciada a partir de um diagnóstico clínico e diferencial correto, isto é, uma correta anamnese, associada a um exame clínico e radiográfico detalhado, possibilita a diferenciação da hipersensibilidade dentinária de outras patologias que acometem os dentes (Faria \& Vilela, 2000). Para tanto, é importante que o clínico tenha conhecimento teórico dos fatores etiológicos da $\mathrm{HD}$, para que não ocorra sub ou superestimação desta condição (Silva et al., 2017).

Classificados quanto ao uso profissional e caseiro, os tratamentos para a hipersensibilidade devem ser prescritos de maneira racional por um profissional. Segundo Rocha et al. (2016), os de uso profissional são produtos que exigem habilidade técnica. Este apresenta a formulação de géis ou aquoso e é mais empregado quando a dor é de maior grau. Os tratamentos caseiros são apresentados no formato de dentifrícios, enxaguantes bucais, entre outros, sendo de fácil uso (Silva et al., 2017).

Os dessensibilizantes são as principais substâncias que possuem como mecanismo de ação a redução da exacerbação da sensibilidade, podendo atuar de maneira oclusiva e/ou neural (Rios et al., 2014). Os dessensibilizantes de ação oclusiva podem agir sobre a alteração do conteúdo dos túbulos dentários por meio de coagulação, pela deposição de proteínas, pela formação e deposição de complexos de cálcio insolúveis e pelo recobrimento da dentina. Os agentes que atuam no segmento nervoso, agem despolarizando as membranas das fibras nervosas, bloqueando, assim, a transmissão de sinais dolorosos ao sistema nervoso central (Ribeiro et al., 2016).

Exemplos de substâncias dessensibilizantes são os dentifrícios. Constituindo umas das primeiras e menos custosas alternativas de indicação profissional para o tratamento da HD, o seu uso é de fácil execução e a aplicação é realizada em casa (Rocha et al., 2016). Apesar de possuir uma composição complexa e industrializada, o mecanismo de ação de cada produto depende do tipo de agente dessensibilizante que o compõe, isto é, podem agir de maneira oclusiva ou interrompendo o estímulo doloroso.

Mesquitas et al. (2009) afirmam que geralmente, as substâncias mais encontradas nos dentifrícios são os sais de potássio (age na despolarização das fibras nervosas impedindo a passagem do estímulo nervoso para o sistema nervoso central); o cloreto de estrôncio e o hidróxido de cálcio que possuem ação oclusiva sobre os túbulos dentinários por meio da deposição de cristais e 
fosfato de cálcio; e a arginina que é um aminoácido natural presente na saliva, promove benefícios no tratamento contra a dor (Querido; Raslan \& Scherma, 2010). A arginina possui estudos que garantem uma vantagem do seu uso quanto ao tratamento duradouro mesmo diante da exposição química, em especial ácida, porém o uso de dentifrícios com essa constituição podem ter um tempo de ação de até 12 semanas, o que se torna uma desvantagem no seu uso (Zado \& Pilatti, 2016).

Uma alternativa usual na clínica é o uso de restaurações de cimento de ionômero de vidro e resina composta para pacientes que além da hipersensibilidade dentinária possuem lesões cavitadas associadas. De acordo com Querido; Raslan \& Scherma (2010), o cimento de ionômero de vidro, quando usado como função restauradora, possui a vantagem de ser hidrofílico e não necessita de ataque ácido previamente. Nesse contexto, independente do o tipo de cimento empregado, todos mostram-se vantajosos em forma protetiva do tecido tubular exposto e na liberação de flúor no meio oral (Godinho; Grippi \& Costa, 2011). Contudo, devido a sua complexa inclusão e por terem menor resistência ao desgaste, à compressão e à tensão, estes são menos utilizados comparados às resinas. $O$ tratamento restaurador em resina composta mostra-se importante recurso a ser aplicado na oclusão de tecido dentinário exposto (Aguiar et al., 2005).

Segundo Xavier, Pinto \& Cavalcanti (2012), as substâncias fluoretadas em contato direto com a superfície mineralizada do dente reagem e precipitam na forma de cristais de fluoreto de cálcio, ocluindo os túbulos. Contudo, quanto ao uso do flúor, os cristais formados a partir da reação química supracitada podem possuir diâmetro menor que os túbulos dentinários, sendo necessário a realização da técnica até a completa obstrução desses (Sousa et al., 2018).

O uso de lasers no Brasil é atualmente uma opção de tratamento para a hipersensibilidade dentinária. Esse possui duas classificações: baixa potência e alta potência. Os lasers de baixa potência possuem ação moduladora, analgésica e antinflamatória, isto é, possibilitam a liberação de hormônios do tipo endorfinas que promovem o bloqueio dos estímulos dolorosos. Nesse aspecto, os lasers de alta potência atuam na desnaturação e coagulação de proteínas, gerando a obliteração dos túbulos dentinários (Figueiredo; Santos \& Batista, 2015).

Segundo Amaral et al. (2012), o tratamento com o uso de laser tem sido viável e eficiente, contudo, a resolução do problema não é definitiva sendo necessário várias aplicações para controle. Além disso, requer conhecimento técnico do profissional e possui alto custo para o paciente. Diante desse cenário, não há diferenças relevantes entre os resultados obtidos entre os dois tipos de lasers. Contudo, a associação dos dois modelos ou o uso isolado desses são significativas entre os resultados obtidos entre os lasers de baixa e alta potência (Hashim et al., 2014).

Arruda et al. (2021) realizou um estudo no qual demonstrou que todos os agentes dessensibilizantes, a base de nitrato de potássio, foram capazes de tratar de maneira eficaz a hipersensibilidade dentinária. A maneira de utilização do nitrato de potássio que mais apresentou resultados satisfatórios, foi na associação de escovação e enxague, duas vezes ao dia durante 2 minutos.

\section{Discussão}

Os tratamentos para HD baseiam-se no uso de agentes dessensibilizantes, flúor, adesivos dentinários, uso de laser, restaurações, cirurgias muco-gengivais e tratamento endodôntico (Zado \& Pilatti, 2016).

De acordo com estudo clássico de Aranha (2003) o material ideal para tratamento de hipersensibilidade dentinária deve ser biocompatível, de fácil aplicação, ter efeito duradouro, permanente e de ação rápida, não ser um irritante pulpar e não alterar a cor da estrutura dental.

Para Kimura et al. (2000) existem duas abordagens para tratamento de alívio da hipersensibilidade dentinária. A primeira abordagem trata-se de interromper a resposta neural dos estímulos de dor pela penetração dos íons de potássio através dos túbulos dentinários para as fibras nervosas, diminuindo a excitação dos nervos. A segunda consiste em bloquear o mecanismo hidrodinâmico através da oclusão dos túbulos abertos. 
Para Aranha et al. (2004) há uma classificação dos agentes dessensibiliantes com a finalidade de tratamento da hipersensibilidade dentinária. São classificados em: 1) agentes anti-inflamatórios; 2) precipitantes de proteínas; 3) agentes de oclusão tubular; 4) selantes de túbulos dentinários (adesivos e resinas) e 5) variados (laser, associado ou não a outros tratamentos).

Segundo Porto, Andrade e Montes (2009) os cremes dentais são os veículos mais comuns para agentes dessensibilizantes, pois são de fácil aplicação, baixo custo e são facilmente encontrados. Os cremes dentais dessensibilizantes agem na obliteração dos túbulos dentinários pela precipitação de fosfato de cálcio na superfície da dentina.

O estudo de Petrou et al. 2009 comprova que cremes dentais contendo arginina e cabonato de cálcio proporcionam um alivio significativo da hipersensibilidade dentinária. Ele relatou o desenvolvimento de uma tecnologia dessensibilizante chamada Pro-Argin, que contém como componentes a arginina, um aminoácido que apresenta um pH entre entre 6,5-7,5, bicarbonato e carbonato de cálcio. Essa tecnologia mostra que esses componentes são capazes de aliviar a hipersensibilidade dentinária através da obliteração e a formação de um "pugle" nos túbulos dentinários. O estudo, demonstra que a arginina e o carbonato de cálcio trabalham em conjunto para acelerar os mecanismos naturais de oclusão dos túbulos e formar uma camada protetora na superfície dentária.

O estudo de Godinho, Grippi e Costa (2011) teve como objetivo avaliar clinicamente a eficácia de dois novos cremes dentais no tratamento dos sintomas causados pela hipersensibilidade dentinária. Avaliaram 86 dentes com lesões cervicais não cariosas em 29 pacientes de ambos os sexos. Os pacientes foram divididos em dois grupos: $\mathrm{G} 1$ - $(\mathrm{n}=42)$ Colgate $®$ Sensitive Pro-Alívio com os seguintes ingredientes ativos: Arginina 8\%, Carbonato de cálcio, Monofluorfosfato de sódio 1.10\% (1450 ppm de Flúor); e G2 - (n = 44) Sensodyne® Rápido Alívio contendo Acetato de estrôncio 8\%, Carbonato de cálcio, Fluoreto de sódio (1040 ppm de Flúor). Para avaliarem a sensibilidade, fizeram o teste evaporativo utilizando-se a seringa tríplice, e os escores de dor foram registrados pela escala visual analógica (VAS) antes e imediatamente após a aplicação tópica do dentifrício. Os resultados demonstraram que os dois cremes dentais avaliados promoveram um alívio imediato da hipersensibilidade dentinária, podendo ser indicados para o tratamento dessa condição. Não houve diferenças significativas entre G1 e G2.

West et al. (1997) apresentou um estudo onde mostra que um creme dental a base de acetato de estrôncio não difere de um creme dental placebo, sendo assim, não promovendo a redução da hipersensibilidade dentinária. Porém, em estudos mais recentes como o de Mason et al. (2010) mostram que o creme dental contendo acetato de estrôncio a $8 \%$ em conjunto com alguns outros ingredientes ativos como por exemplo o fluoreto de sódio e o carbonato de cálcio tem eficácia imediata e duradoura em promover o alivio da sensibilidade dentinária.

Hunghes et al. (2010) corroboram com Mason et al. (2010) e Godinho, Grippi \& Costa (2011) onde comprovam a eficácia dos cremes dentais a base de arginina $8 \%$ e de acetato de estrôncio a $8 \%$, onde relatam que ambos promoveram a reduções significativas na hipersensibilidade dentinária e não havendo diferença significativa entre eles.

Para Clarck e Kevin (2016) além do tratamento caseiro, também há o tratamento de consultório como: materiais resinosos, vernizes fluoretados, oxalatos, hidroxietilmetacrilato, entre outros, que na maioria das vezes agem obliterando os túbulos dentinários expostos.

De acordo com Pandit et al., 2012 o verniz fluoretado é um dessensibilizante que se aplica pincelando a solução sobre regiões que apresentam HD, como por exemplo as lesões cervicais não cariosas. Ele permanece na superfície do dente por meio de interação com a saliva e assim tem uma máxima captação de flúor. O verniz também penetra no tecido dentinário e sela os túbulos dentinários parcial ou completamente (Arends et al., 1997)

Um estudo feito por Guimarães (2015), teve por objetivo avaliar o número de aplicações necessárias para a completa obliteração dos túbulos dentinários utilizando verniz fluoretado, adesivo dentinário, dessensibilizante dentinário e creme dental. Nesse estudo foram utilizados 40 incisivos bovinos, onde foram removidas as porções de esmalte coronário da face vestibular 
(mais plana) de todos os dentes com o auxílio de lixas d'agua de granulações 211Q e 231Q (3M, Sp Brasil) com lixadeira Aropol $2 \mathrm{~V}$ para expor a dentina. Em seguida, removeram as raízes dos dentes a $3 \mathrm{~mm}$ da junção amelo-cementária com disco diamantado de granulação fina e dupla face total e também a porção coronária referente ao terço incisal. Logo após foi realizada a remoção completa de smear layes, evidenciando os túbulos dentinários. Os agentes dessensibilizantes foram aplicados logo após, de acordo com as recomendações dos fabricantes. As amostras foram avaliadas por microscopia eletrônica de varredura de baixo vácuo e foram avaliadas previamente e após o uso dos agentes. A aplicação e a análise foram realizadas até a obliteração total os túbulos dentinários. Tendo então como resultado o verniz fluoretado como o agente dessensibilizante mais eficaz de todos os agentes avaliados, foi necessária apenas uma aplicação para a completa obliteração dos túbulos dentinários, enquanto o adesivo e o creme dental foram necessárias duas e cinco aplicações respectivamente.

Segundo Porto et al., (2009) a combinação de glutaraldeido e hidroxietil metacrilato causa obliteração nos túbulos dentinários através de uma penetração de 50-200 m, e tem comprovação no tratamento da HD. O Gluma Desensitizer contém metacrilato de hidroxietila (HEMA), cloreto de benzalcônio, glutaraldeído e fluoreto e causam uma coagulação no interior das proteínas que ficam nos túbulos dentinários e o metacrilato de hidroxietila causa a formação de marcadores resinosos profundos e oclui os túbulos dentinários. Segundo a literatura o Gluma possui bons resultados no manejo da HD (Davari; Ataei \& Assarzadeh, 2013).

Porém, de acordo com o estudo clínico de Assis et al. (2006), onde 12 sujeitos tiverem 2 dentes tratados, um com Gluma Desensitizer (de acordo com a orientação do fabricante) e o outro com água, não ocorreram diferenças significativas para os dentes testados com Gluma e água em nenhum dos tempos experimentais. Entretanto, para Lopes \& Aranha (2013) a associação de laser e Gluma Desensitizer é uma estratégia de tratamento eficaz que tem efeitos imediatos e ao longo prazo na redução da hipersensibilidade dentinária.

Oxalato é outro dessensibilizante que funciona através da combinação com os íons de cálcio que estão presentes na saliva. Essa combinação oblitera os túbulos dentinários, pois se forma cristais de oxalato de cálcio que são insolúveis e precipitam no interior dos túbulos (Antoniazzi et al., 2014). Além disso, o oxalato oferece resistência ao meio ácido, tornando-se mais durável do que os outros agentes dessensibilizantes (Pashley \& Galloway, 1985).

Porém, de acordo com Miglani, Aggarwal e Ahuja (2010) existe a remoção desses cristais de oxalato de cálcio através da escovação e da dieta ácida, fazendo com que o efeito dessensibilizante do oxalato diminua com o passar do tempo. Mas existe uma maneira de reverter essa condição, condicionando previamente a superfície da dentina, fazendo com que aumente a profundidade de penetração dos cristais de oxalato de cálcio nos túbulos dentinários.

As restaurações cervicais são uma opção mais imediata para o tratamento da hipersensibilidade dentinária (Clark \& Levin, 2016). Quando o esmalte ou a dentina é perdido devido a biocorrosão, tensão ou fricção, a restauração da dentina exposta sela os túbulos dentinários e reduz ou elimina os sintomas da HD (Oliveira, 2018).

Lin et al. (2012) realizou uma revisão sistemática e meta análise onde analisava a eficácia das terapias utilizadas nos consultórios em relação ao placebo para a resolução da HD. Quarenta estudos foram incluídos, a maioria utilizou a escala VAS para mensurar a HD, com um tempo de acompanhamento de até 9 meses. Conclui-se que, em relação à eficácia do tratamento da $\mathrm{HD}$, os produtos em consultório, incluindo o ionômero de vidro foram mais eficazes do que o placebo.

Marto et al. (2019) em sua revisão sistemática comparou quais agentes dessensibilizantes são mais eficazes no tratamento da HD, levando em conta a eficácia e a duração do efeito. Incluiu 74 ensaios clínicos randomizados que utilizavam o método de estimulação evaporativa e VAS. O tempo de acompanhamento ficou definido em: imediato, médio prazo e longo prazo. Concluiu-se que os tratamentos de consultório como glutaraldeído, cimentos de ionômero de vidro e Laser são eficazes na redução imediata da dor associada à HD, permanecendo sua eficácia ao longo do tempo. 
Segundo Nascimento et al. (2020), entre produtos e métodos que se mostram eficazes para o tratamento da HD, estão verniz fluoretado, nanohidroxiapatita (npHA), laser em baixo nível, além de uma terapia conjugada do verniz fluoretado e o laser. Pensando em procedimento restaurador de dentes com HD, primeiro deve-se levar em consideração a remoção dos fatores responsáveis pela causa da lesão. O ionômero de vidro é usado para restaurações de LCNC por sua capacidade de ligação química à dentina e ao esmalte, liberando flúor (Gordan et al., 2014).

Segundo Sobral (2003), as resinas compostas produzem um efeito "tampão", que vai produzir um bloqueio na entrada dos túbulos dentinários, e assim reduz a HD. O seu emprego é recomendado quando a HD dentinária está associada a perda da estrutura dental. Porém Konish et al. (2001) observaram que as resinas compostas, tanto dos sistemas fotoativado quanto do químico, quando sem forramento e sob o ataque ácido dentinário, apresentam um alto risco de sensibilidade pós-operatória. Mas segundo West, Seong e Davies (2015) se aplicadas profissionalmente e corretamente, as resinas compostas são eficazes no tratamento da HD.

Os lasers são um dos tratamentos promissores para a redução da HD, foi demonstrado que os lasers de baixa potência (intensidade), atuam no nível nervoso, e eliminam ou reduzem a sensibilidade, são eles: laser de hélio-neon (He-Ne), laser de arsenieto de gálio e alumínio (GaAlAs) e laser de diodo. Os lasers de alta potência (intensidade) estimulam a produção de dentina terciaria, causando oclusão nos túbulos dentinário. São eles: laser de dióxido de carbono (CO2), laser de cristal de granadaalumínioítrio dopado com neodímio (Nd: YAG), laser de cristal de granada-alumínio-ítrio dopado com érbio (Er: YAG) e laser de cristal de granada-gálio-escândio- ítrio e érbio dopado com cromo (Er,Cr:YSGG) (Kimura et al., 2000).

Segundo Biagi et al. (2016) na última década, os vários tipos de lasers empregados na odontologia, independente do comprimento de onda e protocolo de aplicação, mostraram-se efetivos para a redução ou eliminação da HD.

Mendes et al. (2021) realizaram uma revisão sistemática de estudos publicados de 2016 a 2020 e de acordo com a revisão o emprego do laser, tanto o de baixa ou de alta potência, de maneira geral foi efetivo na grande maioria dos protocolos que foram utilizados nos estudos. Porém ainda não é clara a sua estratégia efetiva a longo prazo. Para Shintome et al. (2007) tanto o laser de baixa quanto o laser de alta intensidade produzem uma diminuição significativamente na hipersensibilidade dentinária.

Kong et al. (2020) realizou uma meta análise afim de comparar diferentes lasers, placebo e nenhum tratamento. Foram inclusos 11 estudos que abordaram sobre os lasers GaAlAs, Nd: YAG, Er: YAG e Er, Cr: YSSG. De acordo com os resultados os lasers Er, Cr: YSGG, Nd: YAG, Er: YAG e GaAlAs tiverem melhores efeitos dessensibilizantes de prazo imediato a longo prazo do que nenhum tratamento, porém não foram melhores que o placebo, não houve diferença significativa. Já Er, Cr: YSGG no efeito imediato demonstrou um efeito melhor do que placebo. E de acordo com o estudo não houve diferenças significativas estatisticamente entre os lasers Er, Cr: YSGG, Nd: YAG, Er: YAG e GaAlAs, tanto no prazo imediato quanto no longo prazo (1 mês). Mas, esse trabalho apresenta um resultado positivo para o uso de terapia a laser, pois os artigos mostraram que os lasers de baixa ou alta potência mostraram uma redução na sintomatologia da HD, tanto no tratamento imediato ou a longo prazo.

Assim como o laser de baixa potência, outro agente dessensibilizante de ação neural é o nitrato de potássio. De acordo com Martins et al. (2012) o seu papel é evitar a transmissão da dor ao sistema nervoso central. O aumento da concentração do potássio extracelular despolariza as membranas das fibras nervosas, impedindo que haja a passagem do estimulo doloroso e consequentemente da hipersensibilidade dentinária.

Silva et al. (2020) demonstrou que a aplicação combinada de nitrato de potássio (agente neural) a $5 \%$ e cloreto de estrôncio (agente obliterador) a 2\% mostraram uma redução significante na Hipersensibilidade dentinária. 


\section{Conclusão}

De acordo com a revisão dos trabalhos contemplados os tratamentos apresentados são eficazes em reduzir a hipersensibilidade dentinária. Uma combinação de instrução de higiene oral, uso de dessensibilizantes caseiros e tratamentos realizados em consultório odontológico, incluindo dessensibilizantes de uso profissional, laser e tratamentos restauradores, são capazes de diminuir e eliminar a hipersensibilidade dentinária. Entretanto, há necessidade de estudos mais complexos para o aumento da qualidade de protocolos eficientes.

Para o sucesso do tratamento, o diagnóstico da hipersensibilidade dentinária deve ser feito de maneira minuciosa, identificando as possíveis causas da dor.

\section{Referências}

Aguiar, F. H. B., Giovanni, E. M., Monteiro, F. H. I., Vilalba, H., Mello, J. J., \& Tortamano, N. (2005). Hipersensibilidade dentinária-causas e tratamentos. Revista de Ciência da Saúde, 23(1),67-71.: https://repositorio.unip.br/wp-content/uploads/2020/12/V23_N1_2005_p67-72-1.pdf

Amaral, S. M., Abad, E. C., Maia, K. D., Weyne, S., Oliveira M. P. R. P. B. \& Tunãs, I. T. C. (2012). Lesões não cariosas: o desafio do diagnóstico multidisciplinar. Arquivo Internacional Otorrinolaringologia, 16(1), 96-102. https://doi.org/10.7162/S1809-48722011000100014

Antoniazzi, R. P., Machado, M. E., Grellmann, A. P., Santos, R. C. \& Zanatta, F. B. (2014). Effectiveness of a desensitizing agent for topical and home use for dentin hypersensitivity: a randomized clinical trial. Am J Dent., 27, 251-257. https://pubmed.ncbi.nlm.nih.gov/25842457/

Aranha, A. C. C. \& Marchi, G. M. (2004). Hipersensibilidade dentinária: Aspectos Gerais e Tratamento. Jornal Brasileiro de Clínica Odontológica Integrada, 8(44),179-184. http://portal.revistas.bvs.br/index.php?search=JBC\%20j.\%20bras.\%20clin.\%20odontol.\%20integr\&connector=ET\&lang=pt

Aranha, A. C., Pimenta L. A. \& Marchi, G. M. (2009). Clinical evaluation of desensitizing treatments for cervical dentin hypersensitivity.Braz Oral Res., 23(3), 333-9. 10.1590/s 1806-83242009000300018

Arends, J., Duscher, H. \& Ruben, J.C. (1997). Penetration of varnishes into demineralised root dentine in vitro. Caries Res, 31, 201-205. 10.1159 / 000262399

Arruda, H. S. Lemos, M. A., Mecozzi, M. L. P., Farias, Z. B. B. M, Prado, A. M. \& Montes, M. A. J. R. (2021) Uso do nitrato de potássio no tratamento da hipersensibilidade dentinária: uma revisão de literatura. Revista Uningá, 58. doi.org/10.46311/2318-0579.58.eUJ3339

Assis, C. A., Antoniazzi, R. P., Zanatta, F. B. \& Rösing, C. K. (2006) Efficacy of Gluma Desensitizer® on dentin hypersensitivity in periodontally treated patients. Braz. oral res. 20 (3). https://doi.org/10.1590/S1806-83242006000300013

Barros, F. C., Antunes, S. A., Figueredo C. M. S. \& Fischer R. G. (2008). Laser de baixa intensidade na cicatrização periodontal. $R$ Ci Med Biol. 7, 85-9. https://doi.org/10.9771/cmbio.v7i1.4362

Biagi R., Cossellu, G., Sarcina, M., Pizzamiglio, I. T. \& Farronato, G. (2016). Laser-assisted treatment of dentinal hypersensitivity: a literature review. Ann Stomatol (Roma), 6, 75-80. 10.11138 / ads / 2015.6.3.075

Clark, D. \& Levin, L. (2016). Non-surgical management of tooth hypersensitivity. Int Dent J., 66, 249-256. 10.1111 / idj.12247

Costa, L. (2007). Prevalência das lesões dentárias não cariosas e sua relação com processos erosivos [Tese]. Universidade de São Paulo, São Paulo, Brasil. https://teses.usp.br/teses/disponiveis/25/25131/tde-09112007-110727/es.php

Davari, A., Ataei, E. \& Assarzadeh, H. (2013). Dentin hypersensitivity: etiology, diagnosis and treatment, a literature review. Journal of dentistry (Shiraz, Iran), 14(3), 136-45. https://pubmed.ncbi.nlm.nih.gov/24724135/

Esteves, C. M. O. A. P. (2010). Lesões cervicais não cariosas [Monografia]. Governador Valadares, MG: Universidade Vale do Rio Doce. https://silo.tips/download/universidade-vale-do-rio-doce-faculdade-de-ciencias-da-saude-curso-de-especializ

Faria, G. J. M. \& Vilela, L. C. (2000) Etiologia e tratamento da hipersensibilidade dentinária em dentes com lesões cervicais não cariosas. Revista de Biociência, 6(70), 21-27. http://revodonto.bvsalud.org/scielo.php?script=sci_nlinks\&ref=415158\&pid=S0004-5276201400010000900004\&lng=es

Figueredo, V. M. G., Santos, R. L., Batista, A. U. D. (2015). Noncarious cervical lesions in occlusion service patients: occlusal aspects and risk factors. Revista Gaúcha de Odontologia, 63(4), 389-396. https://doi.org/10.1590/1981-863720150003000032941

Garone Filho, W. Lesões cervicais e hipersensibilidade dentinária. In: Todescan, F. F., Bottino, M. A. (1996). Atualização na clínica odontológica: a prática da clínica geral. (cap.3, p.35-75) São Paulo: Artes Médicas.

Godinho, C. J., Grippi, M. F. \& Costa, L. C. (2011). Avaliação clínica do uso de dois novos cremes dentais no tratamento da hipersensibilidade dentinária. Revista de Pós Graduação, 18(2),72-78. http://revodonto.bvsalud.org/scielo.php?pid=S0104-56952011000200002\&script=sci_abstract

Gondim, R. C. D. (2011). Hipersensibilidade dentinária de lesões cervicais não cariosas: abordagens terapêuticas no controle da dor. Rev Pesq Saúde, 12(1), 5255. http://www.periodicoseletronicos.ufma.br/index.php/revistahuufma/article/view/938 
Gordan, V. V., Blaser, P. K., Watson, R. E., Mjör, I. A., McEdward, D. L., Sensi, L. G., \& Riley, J. L., (2014). A clinical evaluation of a giomer restorative system containing surface prereacted glass ionomer filler: results from a 13-year recall examination. J Am Dent Assoc., 145 (10), 1036-43. 10.14219/ jada. 2014.57

Grippo, J. O., Simring, M. \& Schreiner, S. (2004). Attrition, abrasion, corrosion and abfraction revisited: a new perspective on tooth surface lesions. Journal American Dentistry Association, 135 (8), 1109-1118. 10.14219 / jada.archive.2004.0369

Guimarães, C. S. R. Efeito de Agentes Dessensibilizantes na Obliteração dos Túbulos Dentinários.(2015). Dissertação (Mestrado em Clínica Odontológica Área de concentração: Dentística) -Faculdade de Odontologia, Universidade Federal do Rio de Janeiro, Rio de Janeiro. http://objdig.ufrj.br/50/teses/m/CCS_M_871304.pdf

Hashim, N. T., Gasmalla, B. G., Sabahelkheir, A. H. \& Awooda, A. M. (2014). Effect of the clinical application of the diode laser (810 nm) in the treatment of dentine hypersensitivity. BMC ResNotes, 14(7), 31. 10.1186/1756-0500-7-31

Hotta, T. H. Marchesan, J. T., Santos, T. M., Silva, M. A. M. R., Silva, R. S., Pécora, J. D. (2006). Uso de laser e placa oclusal na sensibilidade dentinária de bruxômeros. $R G O$, 54(2),195-198.https://pesquisa.bvsalud.org/portal/resource/pt/lil-457173

Hughes, N., Mason, S., Jeffery, P., Welton, H., Tobin, M., O'Shea, C., \& Browne, M. (2010). A comparative clinical study investigating the efficacy of a test dentifrice containing $8 \%$ strontium acetate and $1040 \mathrm{ppm}$ sodium fluoride versus a marketed control dentifrice containing $8 \%$ arginine, calcium carbonate, and $1450 \mathrm{ppm}$ sodium monofluorophosphate in reducing dentinal hypersensitivity. The Journal of clinical dentistry, 21(2), 49-55. Recuperado de : https://pubmed.ncbi.nlm.nih.gov/20669816/

Kimura, Y., Wilder-Smith, P., Yonaga, K. \& Matsumoto, K. (2000). Treatment of dentine hypersensitivity by lasers: a review. J Clin Periodontol, $27,715-721$. https://doi.org/10.1034/j.1600-051x.2000.027010715.x

Kliemann, C. (2002). Lesões cervicais não-cariosas por abrasão (escovação traumática). Journal Brazilian Clinical Odontology, 6(33), 204-209. https://pesquisa.bvsalud.org/portal/resource/pt/lil-336095

Konish, R. N., Leonardo, M. R. \& Bausell, J. (2001). Tratamento da hipersensibilidade dental em abrasão e erosão cervical: efeito de materiais e isolamento de campo. Rev Ciências Odontológicas, 4(4),57-61.

Kong, Y., Lei, Y., Li, S., Zhang, Y., Han, J., \& Hu, M. (2020). Network meta-analysis of the desensitizing effects of lasers in patients with dentine hypersensitivity. Clinical oral investigations, 24(6), 1917-1928. https://doi.org/10.1007/s00784-019-03051-3

Lima, L. M., Humerez Filho, H., Lopes, M. G. K. (2005). Contribuição ao estudo da prevalência, do diagnóstico diferencial e de fatores etiológicos das lesões cervicais não-cariosas. Revista Sul Brasileira de Odontologia, 2(2),18-21. Recuperado de : https://pesquisa.bvsalud.org/portal/resource/pt/biblio-873509

Lin, P. Y., Cheng, Y. W., Chu, C. Y., Chien, K. L., Lin, C. P., \& Tu, Y. K. (2013) In-office treatment for dentin hypersensitivity: A systematic review and network meta-analysis. Journal of Clinical Periodontology, 40(1) 53-64. 10.1111/ jcpe.12011

Lopes, A.O., Aranha, A.C. (2013). Comparative evaluation of the effects of Nd: YAG laser and a desensitizer agent on the treatment of dentin hypersensitivity: a clinical study. Photomed Laser Surg, 31(3),132-8. 10.1089/pho.2012.3386

Lussi, A. (2006). Dental erosion: from diagnosis to therapy. Community Dent Oral Epidemiology, 34(5), 398-399. https://www.researchgate.net/publication/288811641_Dental_erosion_From_diagnosis_to_therapy

Lyttle, H. A., Sidhu, N., \& Smyth, B. (1998). A study of the classification and treatment of noncarious cervical lesions by general practitioners. The Journal of prosthetic dentistry, 79(3), 342-346. https://doi.org/10.1016/s0022-3913(98)70248-3

Marochi, C. \& Queiroz, V. Lesões não-cariosas. In: Gomes, J. (2001). Estética em clínica odontológica. (p. 151-94).

Marsiglio, A. A., Trigueiro, M., Cabezon, P. C., Paula, L. M., Morelli, E. M., Yamaguti, P. M. \& Garcia, F. C. P. (2009). Erosão Dental: da Etiologia ao Tratamento. Ciências Biológicas da Saúde, 11(1), 15-19. https://doi.org/10.17921/2447-8938.2009v11n1p\%25p

Mason, S., Hughes, N., Sufi, F., Bannon, L., Maggio, B., North, M., \& Holt, J. (2010). A comparative clinical study investigating the efficacy of a dentifrice containing $8 \%$ strontium acetate and $1040 \mathrm{ppm}$ fluoride in a silica base and a control dentifrice containing $1450 \mathrm{ppm}$ fluoride in a silica base to provide immediate relief of dentin hypersensitivity. The Journal of clinical dentistry, 21(2), 42-48. https://pubmed.ncbi.nlm.nih.gov/20669815/

Matias, M. N. A., Leão, J. C., Menezes Filho, P. F. \& Silva, C. H. V. (2010). Hipersensibilidade dentinária: uma revisão de literatura. Odontol. Clín.-Cient. (Online), 9(3). http://revodonto.bvsalud.org/scielo.php?script=sci_arttext\&pid=S1677-38882010000300004

Martins, R. S., Macêdo, J. B., Muniz, F. W. M. G., Carvalho, R. S. \& Moreira, M. M. S. M. (2012). Composição, princípios ativos e indicações clínicas dos dentifrícios: uma revisão da literatura entre 1989 e 2011. Journal of the Health Sciences Institute, 30(3), 287-291. https://pesquisa.bvsalud.org/portal/resource/pt/lil-670575

Marto, C. M., Baptista Paula, A., Nunes, T., Pimenta, M., Abrantes, A. M., Pires, A. S., Laranjo, M., Coelho, A., Donato, H., Botelho, M. F., Marques Ferreira, M., \& Carrilho, E. (2019). Evaluation of the efficacy of dentin hypersensitivity treatments-A systematic review and follow-up analysis. Journal of oral rehabilitation, 46(10), 952-990. https://doi.org/10.1111/joor.12842

Mendes, S. T. C., Pereira, C. S., Oliveira, J. L., Santos, V. C. S., Gonçalves, B. B. \& Mendes, D. C. (2021). Tratamento da hipersensibilidade dentinária com laser: revisão sistemática. BrJP., 4(2), 152-60. https://doi.org/10.5935/2595-0118.20210025

Mesquitas, C. R., Almeida, J. C. F., Yamaguti, P. M., Paula, L. M. \& Garcia, F. C. P. (2009). Hiperestesia dentinária: opções de tratamento. Dentística on line, 8 (18), 28-34. http://coral.ufsm.br/dentisticaonline/0813.pdf

Miglani, S., Aggarwal, V., \& Ahuja, B. (2010). Dentin hypersensitivity: Recent trends in management. Journal of conservative dentistry : JCD, 13(4), 218-224. https://doi.org/10.4103/0972-0707.73385 
Nascimento, M. E. S., Júnior, J. R. L. S., Lima, M. V. A., Almeida, N. M. S., Hora, S. L. \& Cabral, L. L. (2020). Etiologia e tratamento da hipersensibilidade dentinária na atualidade: revisão integrativa. Research, Society and Development, 9(8). https://doi.org/10.33448/rsd-v9i8.6192

Oliveira, D. W. D., Lages, F. S., Paiva, S. M., Cromley, J. G., Robinson, P. G. \& Cota, L. O. M. (2018). Cross -cultural adaptation of the Brazilian version of the Dentine Hypersensitivity Experience Questionnaire. Brazillian Oral Research, p. 32-37. https://doi.org/10.1590/1807-3107bor-2018.vol32.0037

Oliveira, D. W. D. (2018). Hipersensibilidade dentinária e qualidade de vida relacionada à saúde bucal: adaptação e validação do dheq-15, e revisão sistemática sobre o impacto do tratamento. (Tese de doutorado). UFMG, Belo Horizonte, Minas Gerais, Brasil. https://repositorio.ufmg.br/bitstream/1843/ODON-B26NLE/1/tese_dhelfeson.pdf

Paiva, G., Nunes, L. De J., Genovese, W. J., Nasr, M. K., Faria Paiva, P. \& Faria Paiva, A. (2003). Preparo com laser Er:YAG de lesões dentais cervicais causadas por abfração, abrasão e/ou erosão. J Bras Dent Estet, 2(5), 44-49. https://www.dtscience.com/wp-content/uploads/2015/10/Preparo-com-Laser-ErYAG-de-Lesoes-Dentais-Cervicais-Causadas-por-Abfracao-Abrasso-e-ou-Erosao.pdf

Pashley, D. H., \& Galloway, S. E. (1985). The effects of oxalate treatment on the smear layer of ground surfaces of human dentine. Archives of oral biology, 30(10), 731-737. https://doi.org/10.1016/0003-9969(85)90185-2

Pandit, N., Gupta, R., \& Bansal, A. (2012). Avaliação comparativa de dois agentes dessensibilizantes comercialmente disponíveis para o tratamento da hipersensibilidade dentinária. Indian Journal of Dental Research: publicação oficial da Indian Society for Dental Research, 23 (6), 778783. https://doi.org/10.4103/0970-9290.111259

Petrou, I., Heu, R., Stranick, M., Lavender, S., Zaidel, L., Cummins, D., Sullivan, RJ, Hsueh, C., \& Gimzewski, JK (2009). Uma terapia revolucionária para a hipersensibilidade dentinária: como os produtos odontológicos contendo $8 \%$ de arginina e carbonato de cálcio atuam para proporcionar alívio eficaz aos dentes sensíveis. The Journal of Clinical Dentistry , 20 (1), 23-31. https://pubmed.ncbi.nlm.nih.gov/19489189/

Pieralisi, F. J. S. (2003). Lesões Cervicais Não-Cariosas / Hipersensibilidade Dentinaria Cervical (Hsdc). (Monografia). Curso de especialização em dentistica restauradora. Associação Brasileira De Odontologia - Secção Santa Catarina- Escola De Aperfeiçoamento Profissional, Santa Catarina, Brasil. http://tcc.bu.ufsc.br/Espodonto224903.PDF

Porto, I. C., Andrade, A. K., \& Montes, M. A. (2009). Diagnosis and treatment of dentinal hypersensitivity. Journal of oral science, 51(3), 323-332. https://doi.org/10.2334/josnusd.51.323

Querido, M. T. A., Raslan, A. S., Scherma, A. P. (2010). Hipersensibilidade dentinária - revisão da literatura. Periodontia, 20(2), 39-46. https://pesquisa.bvsalud.org/portal/resource/pt/lil-642335

Regiani, B. C., Rocha, H. N., Tognetti, V. M., \& Andrade, A. P. de. (2020). Hipersensibilidade dentinária em lesões cervicais não cariosas: etiologia e tratamento. Archives Of Health Investigation, 10(1), 42-48. https://doi.org/10.21270/archi.v10i1.4829

Ribeiro, P. J. T., Araújo, A. M. P., Mafra, R. P., Vasconcelos, M. G. \& Vasconcelos, R. G. (2016). Mecanismos de ação dos recursos terapêuticos disponíveis para o tratamento da hipersensibilidade dentinária cervical. Odontology Clinical Cientific, 15(2), 3-90. http://revodonto.bvsalud.org/scielo.php?script=sci_arttext\&pid=S1677-38882016000200002

Rimondini, L., Baroni, C., \& Carrassi, A. (1995). Ultrastructure of hypersensitive and non-sensitive dentine. A study on replica models. Journal of clinical periodontology, 22(12), 899-902. https://doi.org/10.1111/j.1600-051x.1995.tb01792.x

Rios, A. C. F. et al. (2014). Abrasivos: uma análise de dentifrícios comercializados em Salvador. Revista Bahiana de Odontologia, 5(3),141-152. arquivo pdf.

Rocha, C. S., Prado, M., Simão, R. A., Lima, C. O., Gusman, H. (2016). Efeito de agentes dessensibilizantes na obliteração dos túbulos dentinários - estudo in vitro. Revista Brasileira de Odontologia, 73(4), 272-276. http://revodonto.bvsalud.org/scielo.php?pid=S0034-72722016000400003\&script=sci_arttext

Rösing, C. K., Fiorini, T., Liberman, D. N. \& Cavagni, J. (2009). Dentine hypersensitivity: analysis of selfcare products. Braz Oral Res, 23(Spec Iss 1), 56-63. https://www.scielo.br/j/bor/a/BFKvbzxLtFwthTqfTDrXbPH/?format=pdf\&lang=en

Rother, E. T. Revisão sistemática X revisão narrativa. (2007). Acta paul. Enferm., 20 (2). https://doi.org/10.1590/S0103-21002007000200001

Santos, F. F. C., Lopes, F. F., Thomaz, E. B. A. F., Benatti, B. B., \& Pereira, A. F. V. (2013). Avaliação de Lesões Cervicais Não-Cariosas em Adultos: Estudo Piloto. Pesquisa Brasileira em Odontopediatria e Clínica Integrada, 13 (1), 31-36. https://www.redalyc.org/articulo.oa?id=63727892005

Shintome, L. K., Umetsubo, L. S., Nagayassu, M. P., Jorge A. L. C., Gonçalves S. E. P. \& Torres C. R. G. (2007). Avaliação clínica da laserterapia no tratamento da hipersensibilidade dentinária. Cienc Odontol Bras, 10(1): 26-33. https://doi.org/10.14295/bds.2007.v10i1.417

Silva, L. L. C., Silva, D. F., Rodrigues , R. F., Hora, S. L. \& Lins , F. C. R. (2020). Tratamento químico para hipersensibilidade dentinária - Parte 1 . Research, Society and Development, 9(9). https://doi.org/10.33448/rsd-v9i9.7280

Silva, M. F. et al. (2017). Hipersensibilidade dentinária: desafios para diagnósticos e perspectivas de tratamento. Revista da Associação Paulista de Cirurgiões Dentistas, 71(2), 170-177.

Sobral, M. A. P. Lesões cervicais não cariosas e hipersensibilidade dentinária cervical. In: Garone Netto, N. et al. (2003). Introdução à dentística restauradora (1 ed., p. 265-283). São Paulo: Livraria Santos

Sousa, L. X. de, Cruz, J. H. de A., Melo, W. O. de S., Freire, S. C. P., Ribeiro, E. D., \& Freire, J. C. P. (2018). Abfração dentária: um enfoque sobre a etiologia e o tratamento restaurador. ARCHIVES OF HEALTH INVESTIGATION, 7(2). https://doi.org/10.21270/archi.v7i2.2282

Tolentino, A. B. (2016). Prevalência de LCNC, HD e fatores de risco associados ao estilo de vida de atletas (Dissertação de mestrado). Universidade de São Paulo, São Paulo, Brasil. https://teses.usp.br/teses/disponiveis/23/23152/tde-06032017-100621/pt-br.php 
Research, Society and Development, v. 10, n. 15, e86101522703, 2021

(CC BY 4.0) | ISSN 2525-3409 | DOI: http://dx.doi.org/10.33448/rsd-v10i15.22703

Tonetto, M. R., Dantas, A. A. R., Bortolini, G. F., Fabris, M., Campos, E. A. \& Andrade, M. F. (2012). Hipersensibilidade dentinária cervical: em busca de um tratamento eficaz. Revista Odontológica da Universidade da Cidade de São Paulo, 2(24),190-199. https://repositorio.unesp.br/bitstream/handle/11449/125847/ISSN1983-5183-2012-24-03-190-199.pdf?sequence=1

Watanabe, T., Sano, M., Itoh, K., \& Wakumoto, S. (1991). The effects of primers on the sensitivity of dentin. Dental materials : official publication of the Academy of Dental Materials, 7(3), 148-150. https://doi.org/10.1016/0109-5641(91)90033-u

West, N. X., Addy, M., Jackson, R. J., \& Ridge, D. B. (1997). Dentine hypersensitivity and the placebo response. A comparison of the effect of strontium acetate, potassium nitrate and fluoride toothpastes. Journal of clinical periodontology, 24(4), 209-215. https://doi.org/10.1111/j.1600-051x.1997.tb01833.x

West, N. X., Seong, J., \& Davies, M. (2015). Management of dentine hypersensitivity: efficacy of professionally and self-administered agents. Journal of clinical periodontology, 42 (Suppl 16). https://doi.org/10.1111/jcpe.12336

Xavier, A., Pinto, T., \& Cavalcanti, A. (2017). Lesões Cervicais não cariosas: um panorama atual. Revista de Odontologia da Universidade Cidade de São Paulo, 24(1), 57 - 66. https://doi.org/10.26843/ro_unicid.v24i1.356

Zado, L. N. \& Pilatti, L. G. (2016) Hipersensibilidade dentinária: recentes avanços e tratamentos - revisão de literatura. Brazillian Journal of Periodontology, 26(2), 28-33. https://pesquisa.bvsalud.org/portal/resource/pt/biblio-874882

Zeola, L. F., Soares, P. V. \& Cunha-Cruz, J. (2019). Prevalence of dentin hypersensitivity: Systematic review and meta -analysis. Journal of Dentistry. 